\title{
Variability in the Relationship Between Weight and Wing Length of Anopheles gambiae (Diptera: Culicidae)
}

\author{
JACOB C. KOELLA ${ }^{1}$ AND EDITH O. LYIMO ${ }^{2}$
}

\begin{abstract}
J. Med. Entomol. 33(2): 261-264 (1996)
ABSTRACT The relationship between wing length and body weight of female Anopheles gambiae Giles reared at 3 densities and at 3 temperatures was examined. Although overall, weight was proportional to wing length raised to the 4th power, the relationship within treatments was linear. The slope of the regression line varied significantly among treatments. Therefore, wing length of mosquitoes caught from field populations may not be an adequate measure of body weight if the conditions under which the mosquitoes have developed are not known.
\end{abstract}

KEY WORDS Anopheles gambiae, wing length, body weight

BODY SIZE IS a pivotal trait for mosquitoes, because it has been related to survival (Steinwascher 1982, Hawley 1985, Landry et al. 1988), bloodfeeding behavior (Nasci 1986), reproductive success (Packer and Corbet 1989, Briegel 1990, Bradshaw and Holzapfel 1992), and vector competence (Lyimo and Koella 1992). The best measure of body size is assumed to be dry weight; therefore weight is used in many ecological studies involving associations with body size (Harvey and Pagel 1991). However, the weight of an adult mosquito varies considerably and depends on whether she recently has had a blood or sugar meal. To circumvent this problem, many researchers have used wing length as an indicator of body size. This approach is justified with the observation that wing length generally is correlated closely to a power function of body weight (Christophers 1960, Siegel et al. 1992).

However, wing length can be used as an indicator of body weight only if there is little variability, be it genetical or environmental, in the association between the 2 traits. We investigated the association between wing length and dry weight of female Anopheles gambiae Giles reared at different temperatures and densities. In a previous article, we described wing length and developmental time in detail and have shown that temperature and density affect these traits (Lyimo et al. 1992). Here, we extend the analysis to include dry weight and in particular show that temperature and density affect the association between wing length and dry weight.

\footnotetext{
1 Experimental Ecology, ETH Zentrum NW, 8092 Zürich, Switzerland. Current address: Department of Zoology, University of Århus, 8000 Århus C, Denmark.

2 Ifakara Center, Box 53, Ifakara, Tanzania.
}

\section{Materials and Methods}

The 'Galisua' strain of An. gambiae sensu strictu from Liberia (courtesy of M. Colluzzi, Rome) was reared at 3 temperatures $(24 \pm 0.5,27 \pm 0.3$, and $\left.30 \pm 0.5^{\circ} \mathrm{C}\right)$ and at 3 densities $(100,200$, and 400 larvae per liter). First instars were added to 1 liter of demineralized water in 20 by 10.5 by $8 \mathrm{~cm}$ plastic pans, yielding $0.5,1$, and 2 larvae per square centimeter of surface area. We added $0.2 \mathrm{mg}$ of fish food (Tetramin, Tetrawerke, Melle, Germany) per larva every day until all larvae had pupated. Water was added as necessary to compensate for evaporation.

Pans were checked daily, and dead larvae or pupae were removed. Twenty-four hours after emergence, adults were killed. Females were put individually into gelatin capsules, dried for $48 \mathrm{~h}$ at $40^{\circ} \mathrm{C}$ and then weighed on an electrobalance to the nearest $0.001 \mathrm{mg}$. One wing of each weighed female was glued onto a slide, and its length to the nearest $0.03 \mathrm{~mm}$ was measured with an ocular micrometer from the distal end of the alula to the tip, excluding the fringe scales.

The association between wing length and dry weight was determined as the reduced major axis (Sokal and Rohlf 1981). Model II regression was necessary because both axes were subject to measurement error. The slope of the reduced major axis of 2 traits $\mathrm{X}$ and $\mathrm{Y}$ was the geometric mean of the slope of the regression of $\mathrm{Y}$ on $\mathrm{X}$ and of the reciprocal of the slope of the regression of $\mathrm{X}$ on $\mathrm{Y}$. We checked for linearity of the relationship between wing length and weight using a quadratic regression. When the association between wing length and weight was not linear (that is when the quadratic term was significant) both traits were log-transformed and the parameters of a power function were determined with the regression $\ln ($ weight $)=a+b \ln ($ wing length $)$. 
Table 1. Means and ranges (in parentheses) of adult dry weight at emergence and wing lengths at each combination of temperature and density

\begin{tabular}{cccc}
\hline \hline Density & Temp & Wt, mg & Wing length, mm \\
\hline 100 & 24 & $0.27(0.17-0.49)$ & $2.84(2.60-3.32)$ \\
100 & 27 & $0.28(0.16-0.45)$ & $2.87(2.61-3.22)$ \\
100 & 30 & $0.20(0.13-0.32)$ & $2.64(2.41-2.92)$ \\
200 & 24 & $0.27(0.16-0.45)$ & $2.92(2.68-3.19)$ \\
200 & 27 & $0.26(0.15-0.47)$ & $2.83(2.51-3.10)$ \\
200 & 30 & $0.21(0.14-0.30)$ & $2.67(2.39-2.90)$ \\
400 & 24 & $0.27(0.16-0.49)$ & $2.99(2.76-3.22)$ \\
400 & 27 & $0.25(0.15-0.44)$ & $2.82(2.44-3.17)$ \\
400 & 30 & $0.23(0.12-0.42)$ & $2.73(2.44-3.20)$ \\
\hline
\end{tabular}

The effect of temperature and density on the relationship between wing length and weight was determined by an analysis of variance (ANOVA) with wing length as the dependent variable, temperature and density as main effects, and body weight as a covariate. The interactions of the weight with treatment parameters in determining wing length showed whether the slopes of wing length on weight differed among treatments. The analyses were performed with the program JMP (SAS Institute 1994).

\section{Results and Discussion}

We analyzed 1,668 mosquitoes. Adult dry weight at emergence ranged from 0.12 to $0.5 \mathrm{mg}$ with a mean of $0.25 \mathrm{mg}$; wing length ranged from 2.4 to $3.3 \mathrm{~mm}$ with a mean of $2.83 \mathrm{~mm}$. The means and ranges of weight and wing length for each treatment are shown in Table 1.

Overall, weight was associated closely with wing length (Fig. 1), but the relationship was nonlinear. The log-transformed variables gave a regression coefficient of $2.95\left(r^{2}=0.52, P<0.001\right)$ for $\ln$ (weight) on $\ln$ (wing length) and a coefficient of $0.18\left(r^{2}=0.52, P<0.001\right)$ for $\ln$ (wing length) on $\ln$ (weight). The slope of the reduced major axis was $\sqrt{2.95 / 0.18}=4.08$ with the $95 \%$ CL of 3.90 and 4.26 .

In contrast, there was no evidence of a nonlinear association within treatments. Therefore, untransformed data were used to compare the relationship between weight and wing length within treatments and the differences of the relationship among treatments. In the ANOVA (Table 2), the interaction of temperature and wing length was significant, indicating that the relationship between weight and wing length differed among temperatures. The significant 3-way interaction showed that density also affected this relationship. The slopes of the major axis between weight and wing length ranged from $0.30 \mathrm{mg} / \mathrm{mm}$ at $30^{\circ} \mathrm{C}$ and 100 larvae per liter to $0.67 \mathrm{mg} / \mathrm{mm}$ at $24^{\circ} \mathrm{C}$ and 400 larvae per liter (Fig. 2).

The linear relationship within treatments was not a result of sampling points from a limited portion of the curve (Fig. 3). In most of the treatments, wing length and weight varied over a large

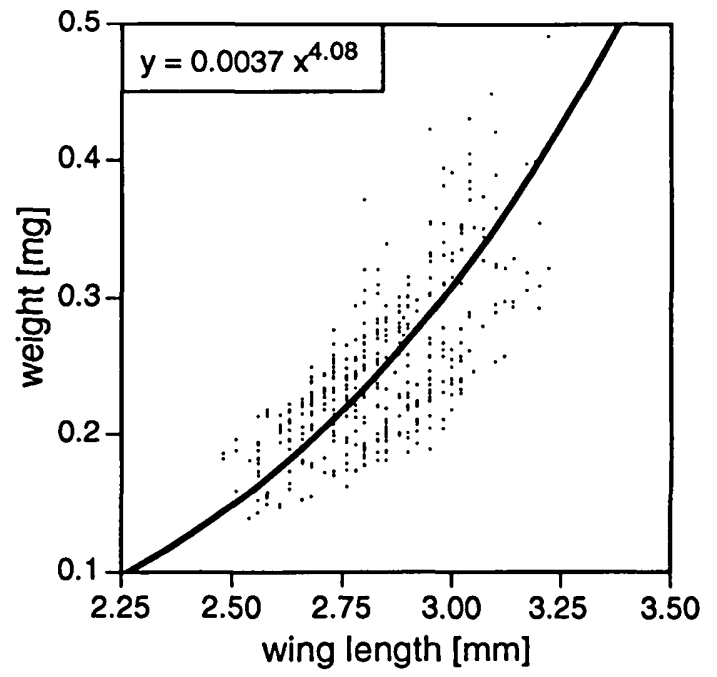

Fig. 1. Association between dry weight and wing length of mosquitoes reared in 9 treatments differing in temperature and density. Each dot represents an individual mosquito; a random subset of $25 \%$ of the total sample is plotted. The line represents the major axis of the logtransformed values and follows the equation shown in the graph.

portion of the total range observed. Furthermore, the associations within treatments were almost always steeper than the overall association would suggest, in particular with small body sizes and at the lowest temperature.

The linear trend within treatments indicated that the curvilinear pattern may be a sampling artifact. Pooling mosquitoes reared under different environmental conditions, and thus with different relationships between weight and wing length, may lead to an apparently nonlinear relationship with an observed slope smaller than the true relationship within treatments.

That the relationship between weight and wing length should be considerably less than a cubic relationship is suggested by aerodynamic theory. The lift achieved by flapping wings increases propor-

Table 2. Results of an analysis of covariance of dry weight of adult females with density and temperature as main effects and wing length as the covariate

\begin{tabular}{lrrr}
\hline \hline \multicolumn{1}{c}{ Source } & $\begin{array}{c}\text { Degrees } \\
\text { of } \\
\text { freedom }\end{array}$ & $\begin{array}{c}\text { Sum of } \\
\text { squares }\end{array}$ & $F$ ratio \\
\hline Density & 2 & 0.001 & $0.5 N S$ \\
Temp & 2 & 0.047 & $16.2^{* * *}$ \\
Density*Temp & 4 & 0.062 & $10.7^{* * *}$ \\
Wing length & 1 & 0.239 & $165.7^{* * *}$ \\
Density*Wing length & 2 & 0.000 & 0.1 NS \\
Temp*Wing length & 2 & 0.046 & $16.0^{* * *}$ \\
Density*Temp*Wing length & 4 & 0.064 & $11.0^{* * *}$ \\
Error & 1,650 & 2.377 & \\
\hline
\end{tabular}

${ }^{a}$ The significance levels are given as NS for $P \geq 0.5$ and *** for $P<0.001$. 


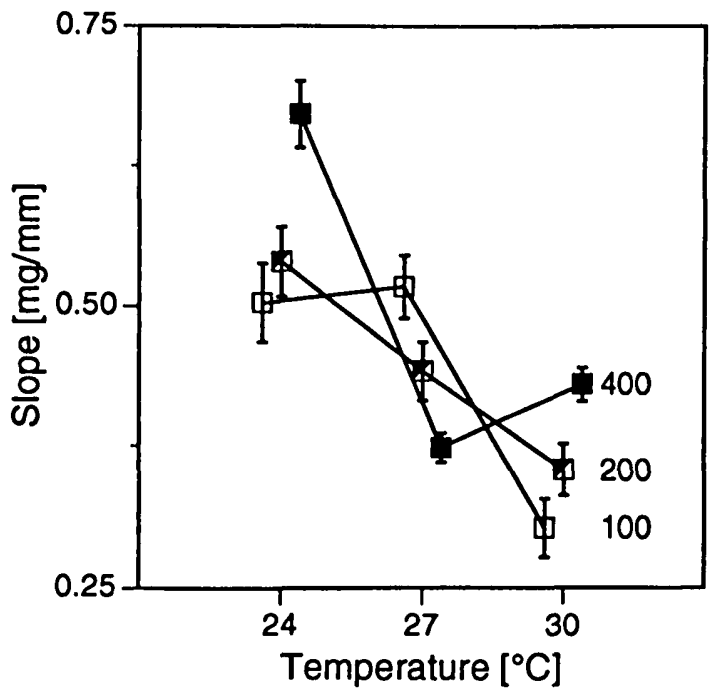

Fig. 2. Slopes of the linear associations between weight and wing length. The mosquitoes from each treatment were analyzed separately; within each treatment the association was linear. The squares show the best estimate of the slope calculated at different densities (solid squares: 400 larvae, half-solid squares: 200 larvae; open squares: 100 larvae). Vertical bars show the standard errors of the slopes.

tionally to the area of the wings and to the amplitude of the wing stroke (Brodsky 1994). Thus, if the wing stroke and the shape of the wings are constant, one would expect a quadratic relationship between weight and wing length. If wing stroke increases with the length of a wing, maintaining lift would require less than a quadratic relationship. To our knowledge, there is no study showing an association between length and wing stroke. However, this association is suggested by the observation that female mosquitoes, which are generally larger than males, have an almost 2-fold larger wing stroke than males (Brodsky 1994).

From a more practical viewpoint, because the relationship between wing length and weight differs among treatments, the interpretation of data from field collections of mosquitoes is difficult. At the least, the standard error of the predicted body weight will become prohibitively large, if the relationship of wing length and weight varies among environments and this variability is not taken into account. Perhaps this may explain why the fieldcollected mosquitoes showed the lowest coefficient of correlation of the 4 studies reviewed by Siegel et al. (1992) and why, in the same review, the slope of the relationship differed between field-collected and laboratory-reared Culiseta melanura.

In conclusion, the wing length of field mosquitoes may not be an adequate measure of body weight if the conditions under which the mosquitoes have developed are not known.

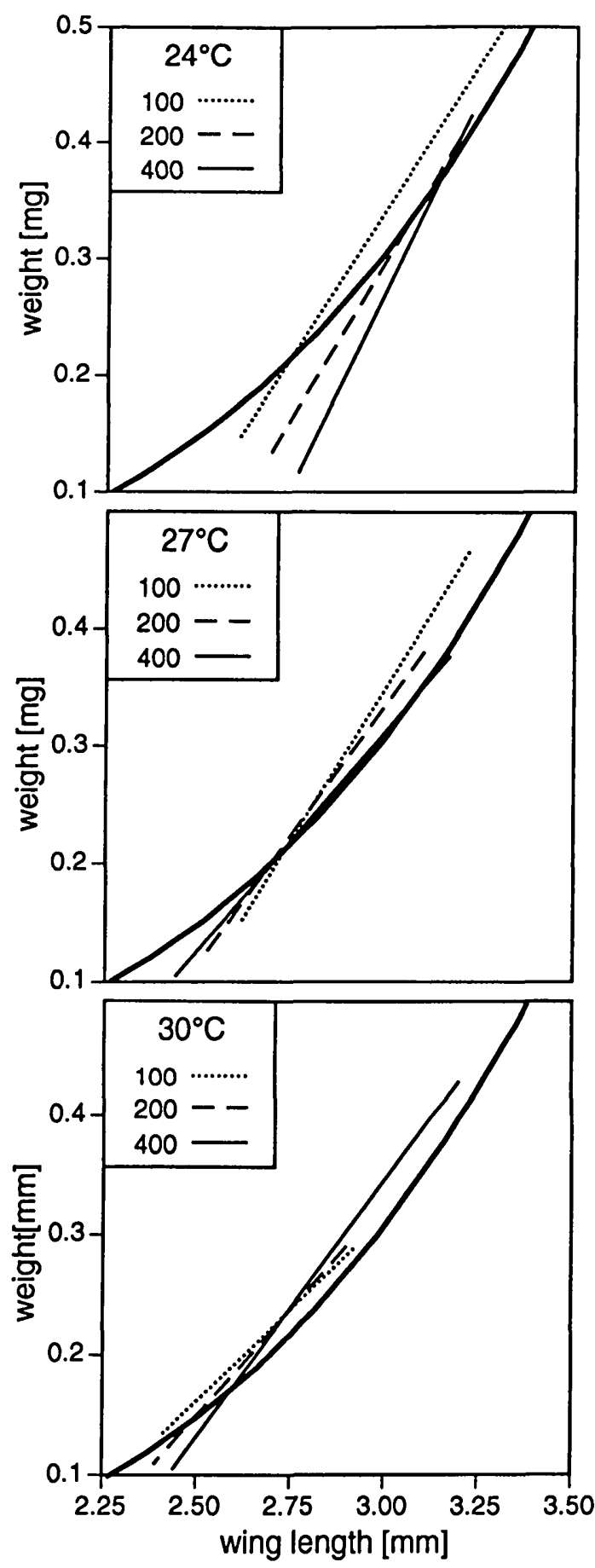

Fig. 3. Association between dry weight and wing length of mosquitoes reared at 24,27 and $30^{\circ} \mathrm{C}$. In each panel, the thick, curved line shows the overall association shown in Fig. 1, and the thin, straight lines show the linear association calculated within each density. The lines are drawn from minimum to maximum wing length and thus show the variation of wing length within each treatment. 


\section{Acknowledgments}

We thank Phil Agnew and 2 anonymous reviewers for helpful comments on the manuscript. The experiments were supported by the Directorate General for International Cooperation of the Government of the Netherlands. The study is part of the Kilombero Malaria Project, a joint project of the Tanzanian, Swiss, and Dutch governments and of the World Health Organization.

\section{References Cited}

Bradshaw, W. E., and C. M. Holzapfel. 1992. Reproductive consequences of density-dependent size variation in the pitcherplant mosquito, Wyeomyia smithii (Diptera: Culicidae). Ann. Entomol. Soc. Am. 85: 274-281.

Briegel, H. 1990. Fecundity, metabolism, and body size in Anopheles (Diptera: Culicidae), vectors of malaria. J. Med. Entomol. 27: 839-850.

Brodsky, A. K. 1994. The evolution of insect flight. Oxford University Press, Oxford, UK.

Christophers, S. R. 1960. Aedes aegypti (L.): the yellow fever mosquito. Its life history, bionomics and structure. Cambridge University Press, Cambridge, UK.

Harvey, P. H., and M. D. Pagel. 1991. The comparative method in evolutionary biology. Oxford University Press, Oxford, UK.

Hawley, W. A. 1985. The effect of larval density on adult longevity in a mosquito, Aedes sierrensis: epidemiological considerations. J. Anim. Ecol. 54: 955964.
Landry, S. V., G. R. De Foliart, and D. B. Hogg. 1988. Adult body size and survivorship in a field population of Aedes triseriatus. J. Am. Mosq. Control Assoc. 4: 121-128.

Lyimo, E. O., and J. C. Koella. 1992. Relationship between body size of adult Anopheles gambiae s.l. and infection with the malaria parasite Plasmodium falciparum. Parasitology 104: 233-237.

Lyimo, E. O., W. Takken, and J. C. Koella. 1992. Effect of rearing temperature and larval density on larval survival, age at pupation and adult size of Anopheles gambiae. Entomol. Exp. Appl. 63: 265271.

Nasci, R. S. 1986. The size of emerging and host-seeking Aedes aegypti and the relation of size to bloodfeeding success in the field. J. Am. Mosq. Control Assoc. 2: 61-62.

Packer, M. J., and P. S. Corbet. 1989. Size variation and reproductive success of female Aedes punctor (Diptera: Culicidae). Ecol. Entomol. 14: 297-309.

SAS Institute. 1994. JMP statistics and graphics guide. SAS Institute, Cary, NC.

Siegel, J. P., R. J. Novak, R. L. Lampman, and B. A. Steinly. 1992. Statistical appraisal of the weightwing length relationship of mosquitoes. J. Med. Entomol. 29: 711-714.

Sokal, R. R., and F. J. Rohlf. 1981. Biometry, 2nd ed. Freeman, San Francisco.

Steinwascher, K. 1982. Relationship between pupal mass and adult survivorship and fecundity for Aedes aegypti. Environ. Entomol. 11: 150-153.

Received for publication 8 February 1995; accepted 28 July 1995. 